\title{
A novel rapid (20-minute) IL-6 release assay using blood mononuclear cells of patients with various clinical forms of drug induced skin injuries
}

\author{
Joseph M Baló-Banga ${ }^{1 *}$, Katalin Schweitzer ${ }^{2}$, Susan Lakatos ${ }^{2}$ and Sándor Sipka ${ }^{3}$
}

\begin{abstract}
Background: IL-6 is a pro-inflammatory cytokine which has many well-defined effects. Its synthesis and release from mononuclear cells of drug-sensitized patients was related before to in vitro drug-allergy diagnostics but has not yet been studied in detail.

Methods: The specific release of preformed IL-6 from peripheral blood mononuclear cells (PBMC) after 20 minutes incubation with $0.15-0.5 \mu \mathrm{M}$ of pure drugs was measured in two groups of drug-allergy suspected donors (159) and respective controls (48). IL-6, TNF-alpha, IL-2, IL-4, IFN-gamma have been measured from cell supernatants by ELISA or by cytometric bead assay. Epicutaneous, intradermal and systemic provocation tests were performed to prove or disprove culprit substances (203 in vivo against 482 in vitro tests). T-test (paired and unpaired); chi2 contingency table; Z statistics and McNemar's test were used to evaluate results.

Results: Concanavalin A as positive control released IL-6 from PBMC in linear concentration and exponential time dependent fashion (up to 60 minutes) pointing to the existence of a preformed pool of this cytokine.

Preformed IL-6 released at any of 4 standard drug dilutions tested, above $50 \%$ over their diluents' levels significantly correlated with the patients' history on drug-induced hypersensitivity symptoms and with in vivo tests.

Sensitivity of $85.4 \%$ and specificity of $82.4 \%$ of the IL-6 release assay were found. The $20^{\prime}$ drop in release of TNF-alpha had no diagnostic importance; it has accompanied increased IL-6 release. IL-2, IL-4 and IFN-gamma were undetectable in 20 minutes supernatants. IL-6 release depended on the clinical phenotype but not on the eliciting drug(s) in the molecular mass range of 76-4000 Da. Reactivity of mononuclear cells at the lowest or at multiple drug test concentrations reflected clinical severity per diagnoses and according to area of skin involvement.
\end{abstract}

Conclusion: This rapid test is applicable to detect a wide scale of drug hypersensitivity.

Keywords: IL-6, TNF-alpha, T-lymphocytes, Drug-induced skin injury, Adverse drug reactions, Preformed cytokines' release

\section{Background}

It is generally accepted that about $20 \%$ of all adverse drug reactions (ADR) are immunologically mediated [1,2]. The majority of these reactions has skin manifestations [3]. The diversity of humoral and cellular mechanisms motivated Pichler to study the different T-cell subsets at certain well-defined clinical pictures. In addition to hapten and prohapten presentation of small molecular drugs the concept of pharmacological

\footnotetext{
* Correspondence: balmat05@freemail.hu

${ }^{1}$ Department of Dermatology, Medical Center of Hungarian Defense Forces, Podmaniczky u. 109-111, Budapest H-1062, Hungary

Full list of author information is available at the end of the article
}

interaction ( $\mathrm{p}-\mathrm{i})$ has emerged [4]. This concept explains for the rapid elicitation of generalized symptoms due to the binding of unchanged drugs to TCR and MHC resulting in activation of mediators and cytokines. Studies were designed to identify and exploit the measurement of those cytokines in search for culprit drugs. These tests have measured de novo synthesized molecules from cultivated cells' supernatants $[2,5,6]$.

IL-6 a 22-27 kDa peptide is involved in numerous cellular and molecular mechanisms of inflammation including $\mathrm{T}$ and $B$ cell activation and synthesis of acute phase proteins by hepatocytes. IL-6 binding to its membrane-bound receptor (IL-6R) 
results in signal transduction [7]. IL-6 type cytokines bind to membrane receptors activating both the JAK/STAT and the MAPK cascades [8].

In earlier studies we and others found that short term ex vivo incubation of PBMCs with standard dilutions of sensitizing drugs has changed the chromatin structure of lymphocytes in a specific manner [1,9]. After a 20-minute incubation with the offending drug release of IL- $1 \alpha$, IL- $1 \beta$, IL-6 cytokines could be measured concomitant with the structure change of chromatin. Chromatin "relaxation" measured by morphometry correlated best with the prompt release of IL-6 [9]. Our preliminary results on 45 ADR suspect patients with five controls were promising [10]. Lochmatter et al. [2] cultivated PBMCs of control donors and of patients with well defined drug allergies for 24-72 hours with aminopenicillins or sulphonamides according to their histories. These PBMCs have shown significant IL-6 release only in AMX sensitive patients. Sixteen other cytokines/chemokines were tested simultaneously as well, out of them IL-5, IFN- $\gamma$, IL-13 and IL-2 seemed to be suitable only in combination for diagnostic purposes.

The aim of the present study was to limit the plethora of measurements to a single cytokine, namely to IL-6 and standardize the sensitivity and specificity of the procedure. Of note, preformed cytokines were not known to operate in mononuclear cells contrary to eosinophils [11] and mast cells [12]. Furthermore, we aimed to demonstrate that early IL-6 release is specific of the drug causing immune-mediated reactions, and it does not depend on the type or structure of drug or on the phenotype of the skin allergic reactions.

\section{Methods}

\section{Patients and controls}

Patients were seen and treated by our group at the Department of Dermatology or as out-patients in the ADR Clinic of the Military Hospital in Budapest. Ninety eight patients with suspected drug hypersensitivity were studied between 2007 and 2011. Both immediate and delayed type allergies were represented (Table 1). There were 80 women and 18 men, their mean age was $49.9 \pm 18.9$ (SD). The patients fell into definitive (46\%), probable $(20 \%)$, possible $(21 \%)$, not related $(11 \%)$, and impossible (2\%) categories as defined by Karch and Lasagna [13]. In 24 control subjects the drugs as offending substances could be ruled out (categories impossible or not related $=98 \%$ ). These groups together were marked as "Test $A$ ". Tests were carried out in a currently symptom-free state as usual between 4 weeks and 1 year after cessation of therapy. Between 2005 and 2007 sixtyone patients and 24 control subjects were seen and tested under identical conditions and selection criteria. In this group there were 49 women and 12 men, mean age was $52.4 \pm 17.9$ (SD). According their history $45 \%$ were

Table 1 Distribution of clinical manifestations of drug hypersensitivity from "Test $A$ " group (values are given in \% of cases)

\begin{tabular}{|c|c|c|c|c|c|}
\hline & & \multicolumn{4}{|c|}{ IL-6 release pattern } \\
\hline \multicolumn{2}{|c|}{ Phenotypes } & $\begin{array}{l}\text { with peak at } 0.15 \mu \mathrm{M} \text {, } \\
\qquad(\mathrm{n}=37 \text { tests) I }\end{array}$ & $\begin{array}{l}\text { with peak at } 0.35 \mu \mathrm{M} \text {, } \\
\qquad(\mathrm{n}=67 \text { tests) }\end{array}$ & $\begin{array}{l}\text { with peak at } 0.5 \mu \mathrm{M} \text {, } \\
\quad(\mathrm{n}=38 \text { tests })\end{array}$ & $\begin{array}{l}\text { with } 2 \text { or more positivities } \\
\text { ( } n=65 \text { tests) IV }\end{array}$ \\
\hline 1 & Generalized urticaria $\pm \mathrm{ANO}^{2}$ & 17 & 18 & 11 & 18 \\
\hline 2 & Systemic Anaphylaxis \pm ANO & 23 & 24 & 24 & 28 \\
\hline 3 & DRESS ${ }^{3}$ (culprit/non-culprit ${ }^{*}$ ) & 3 & 0 & $3^{*}$ & 0 \\
\hline 4 & Generalized MPE (>18\%) $^{4}$ & 14 & 16 & 8 & 20 \\
\hline 5 & Localized MPE (<18\%) & 6 & 4 & 8 & 5 \\
\hline 6 & Disseminated fixed drug eruption & 1 & 2 & 0 & 0 \\
\hline 7 & Erythema multiforme & 0 & 2 & 0 & 2 \\
\hline 8 & Asthma, severe itch & 1 & 0 & 0 & 0 \\
\hline 9 & Generalized disseminated dermatitis & 9 & 2 & 11 & 8 \\
\hline 10 & Small patchy urticaria & 3 & 9 & 8 & 5 \\
\hline 11 & Localized ANO & 14 & 17 & 18 & 8 \\
\hline 12 & Leg dermatits \pm purpurae & 0 & 3 & 3 & 5 \\
\hline 13 & Circumscribed vesiculae & 3 & 0 & 3 & 0 \\
\hline 14 & $\begin{array}{l}\text { Erythema annulare centrifugum/E. } \\
\text { nodosum }\end{array}$ & 6 & 3 & 3 & 1 \\
\hline
\end{tabular}

${ }^{2}$ ANO- angioneurotic edema.

${ }^{3}$ DRESS -drug rash with eosinophilia and systemic symptoms.

${ }^{4}$ MPE- maculopapular exanthema.

*non-culprit drug representation of a single case.

Boldface number in column II marks culprit. 
definitively, $19 \%$ probably, $19 \%$ possibly allergic and $17 \%$ fell into the not related category. None were marked as "impossible". Their matching control group comprised 20 women and 4 men Out of 22 (92\%) 14 persons belonged to the impossible and 8 persons to not related symptoms revealed while 2 persons were possibly allergic. These groups were designated as "Test $B$ ". Assay conditions were different for the two groups. All gave their informed consent and the study was approved by the Ethical Committee of the Medical Center of the Hungarian Defense Forces.

\section{In vitro tests}

\section{Drugs and mitogens}

Non-toxic (final) drug concentrations were used in each test series $0.15 ; 0.25 ; 0.35$ and $0.50 \mu \mathrm{M}$, prepared freshly from pure substances or diluted from sterile injections or other suitable liquid drug formulations. The molecular masses of drugs investigated varied between 76 (Propylene glycol) and $4000 \mathrm{Da}$ (Enoxaparin sodium). The pure drugs selected according to the patients' history were either gifts of certain pharmaceutical companies or had been purchased from LGC Standards GmbH (Wesel, Germany). To obtain in vitro positive controls the cells were stimulated either with PHA-P (PHA $168 \mu \mathrm{g} / \mathrm{ml} ; \mathrm{PHA}_{2} 335 \mu \mathrm{g} / \mathrm{ml}$, Sigma-Aldrich Co.) or with Con A (Sigma-Aldrich, type 6) tested at 5 to $300 \mu \mathrm{g} / \mathrm{ml}$ concentrations.

\section{Separation of PBMC}

Was done by using Ficoll-Paque ${ }^{\mathrm{TM}}$ (Amersham, Biosciences) as described [14] and washed twice with PBS containing $2 \mathrm{mM}$ of EDTA and $0.5 \% \mathrm{w} / \mathrm{v}$ of BSA. The cells were then re-suspended in modified Dulbecco's MEM [15] containing $100 \mathrm{mM} \mathrm{NaCl}, 24 \mathrm{mM} \mathrm{KCl}, 10-10 \mathrm{mM} \mathrm{CaCl} 2$ and $\mathrm{MgCl} 2$, and $11 \mathrm{mM}$ glucose, pH: 7.2 (Test A incubation medium). In earlier experiments a different MEM solution was used containing $145 \mathrm{mM} \mathrm{NaCl}, 21 \mathrm{mM} \mathrm{KCl}$, and 0.7- $0.7 \mathrm{mM}$ $\mathrm{CaCl} 2$ and $\mathrm{MgCl} 2$ and $11 \mathrm{mM}$ glucose, $\mathrm{pH}$ : 7.2 (Test $B$ incubation medium). The incubation of $1.1 \times 10^{6} / \mathrm{ml}$ cells without any plasma or serum was carried out in $450 \mu \mathrm{l}$ aliquots for $20 \mathrm{~min}$ at $37^{\circ} \mathrm{C}$ with drugs or mitogens dissolved in $50 \mu \mathrm{l}$ of solvent. The incubation was terminated by placing the tubes into crushed ice and then the fluid was centrifuged at 30-50 $\times$ g for $6 \mathrm{~min}$. The water-clear supernatants were carefully removed and kept frozen at $-80^{\circ} \mathrm{C}$ until cytokine determinations.

\section{Detection of IL-6 in the cell-free supernatants}

IL-6 was determined in the cell-free supernatants by solid phase immunoassay (Diagnosticum Ltd., Hungary) according to the manufacturer's instructions, as described earlier [16]. In addition, both incubations with polyclonaland monoclonal anti-IL-6 antibodies were performed under mild shaking at $37^{\circ} \mathrm{C}$ for $60 \mathrm{~min}$. The calibration curve was linear between 10 and 700 pg/ml IL-6 concentrations $(0.951<\mathrm{R} 2<0.988)$. O.D. values falling below or above this range were extrapolated.

Cytotoxicity measurements were performed on selected cell-free supernatants using the automated (Roche Modular T-800) determination of LDH.

Simultaneous Detection of IL-2, IL-4, IL-6, IL-10, TNF- $\alpha$ and IFN- $\gamma$ was performed with the BD-CBA Human Th1/ Th2 Cytokine Kit II according to the manufacturer's instruction (Becton Dickinson, Franklin Lakes, NJ, USA). Briefly, $50 \mu \mathrm{l}$ of mixed human Th1/Th2 cytokine capture beads and $50 \mu \mathrm{l}$ of phycoerythrin labeled detection reagent were incubated either with $50 \mu$ l of each test sample or with $50 \mu \mathrm{l}$ of the human Th1/Th2 cytokine standard dilutions for 3 hours at room temperature in dark. After a brief washing $(200 \times \mathrm{g}, 5 \mathrm{~min})$, samples were run on a BDFACS Array flow cytometer. Data acquisition and analysis were performed with the $\mathrm{BD}^{\mathrm{Tm}} \mathrm{CBA}$ software.

\section{In vivo tests}

Drug patch tests were done either with $10 \% \mathrm{w} / \mathrm{w}$ pure substances, or less frequently, with $30 \% \mathrm{w} / \mathrm{w}$ ground powder of tablets in petrolatum. Curatest ${ }^{\mathrm{Tm}}$ (Brial $\mathrm{GmbH}$, Germany) adhesive chambers were used. Occasionally $5-10 \% \mathrm{w} / \mathrm{v}$ solutions in distilled water were prepared. Results were read after $20 \mathrm{~min}, 48 \mathrm{hrs}, 72-96 \mathrm{hrs}$. Intradermal tests were prepared under sterile conditions. Pure drug substances or injection formulations (eye drops) were diluted in 2 steps to obtain $1 \times 10^{-3} \mathrm{M}$ solutions in PBS. Water insoluble substances were first dissolved in DMSO and diluted further with PBS to obtain the desired concentrations. The concentration of DMSO never exceeded $1 \% \mathrm{v} / \mathrm{v}$. Negative (diluent) and positive controls (Histamine $0.1 \mathrm{mg} / \mathrm{ml}$ ) were included with all tests. Injections $(0.04 \mathrm{ml})$ were placed in the volar skin of forearm. Results were recorded at $20 \mathrm{~min}, 90 \mathrm{~min}$ and $24 \mathrm{hrs}$. Positivity was only accepted if $10^{-3} \mathrm{M}$ concentration gave $>3 \mathrm{~mm}$ papules/wheals increasing in time with or without a red halo. Any skin reactions obtained only at higher than $10^{-3} \mathrm{M}$ of drugs or additive substances were considered as "irritative".

Drug provocation tests were performed under conditions set by the ENDA and by the EAACI group on drug hypersensitivity [17]. Incremental doses were given orally $[1,17]$ or subcutaneously under strict control (with emergency room coverage) over 3 hrs. in the ward, followed by a 24 hour phone contact. The tests were performed parallel to in vitro results even after severe reactions or in doubtful cases to differentiate between hypersensitivity and e.g. vagal reaction due to local anaesthetics. Positivity was accepted if skin or systemic symptoms arose (mainly within the close observation period). 


\section{Data analysis}

Statistical significance was determined by the $t$-test for both paired and unpaired data. For analysis of the morbidity rates the $\chi^{2}$ and Z-statistics were employed. A p $<0.05$ value was considered to be statistically significant. Determination of diagnostic efficacy including specificity and sensitivity related to in vivo exposures were generated by McNemar's test.

\section{Results}

The numbers of complete tests in the two groups (with Test $A$ and Test B solutions) are shown in Table 2. The total number of all in vitro diagnostic test series based on IL-6 release was 482. A test was considered positive if the concentration of IL-6 in the supernatant of PBMCs incubated with the drug was higher by $50 \%$ than in its control counterpart at any concentration. Cases where increase of IL-6 was exactly $50 \%$ at any concentration were considered as doubtful.

\section{IL-6 release caused by positive controls (Figure 1)}

The dose response of mononuclear cells to the mitogen Con A was linear between $0-20 \mu \mathrm{g} / \mathrm{ml}$. The time course experiments have shown that the IL- 6 release is almost complete by the $20^{\text {th }}$ min of Con A stimulation. Five $\mu \mathrm{g} / \mathrm{ml}$ was used as positive control. However, much higher doses of PHA-P were needed. Two concentrations, $168 \mu \mathrm{g} / \mathrm{ml}$ $\left(\mathrm{PHA}_{1}\right)$ and $337 \mu \mathrm{g} / \mathrm{ml}\left(\mathrm{PHA}_{2}\right)$ were tested and $\mathrm{PHA}_{1}$ was used. In time course experiments a plateau was reached between 10 and 50 minutes of incubation which declined thereafter (data not shown in details).

\section{Table 2 Summary of the tested groups}

\begin{tabular}{lcc}
\hline Groups & $\begin{array}{c}\text { "Test } \boldsymbol{A}^{\text {" }} \\
\text { solution }\end{array}$ & $\begin{array}{c}\text { "Test } \boldsymbol{B}^{\text {" }} \\
\text { solution }\end{array}$ \\
\hline Number (N) of controls & 24 & 24 \\
N of tests in controls & 50 & 49 \\
N of negative tests in controls & 48 & 48 \\
Doubtful and positive tests in control group & 2 & 1 \\
N of suspect patients & 98 & 61 \\
N of tests in group of suspect patients & 266 & 121 \\
Positive tests in group of suspect patients & 151 & 32 \\
Negative tests in group of suspect patients & 113 & 87 \\
Doubtful tests in group of suspect donors & 2 & 2 \\
(Test/person) for control group & 2.1 & 2.1 \\
(Test/person) for suspect patients & 2.7 & 1.9 \\
Total N of tests & 316 & 166
\end{tabular}

"Test A": $100 \mathrm{mM} \mathrm{NaCl}, 24 \mathrm{mM} \mathrm{KCl}, 10 \mathrm{mM} \mathrm{CaCl} 2,10 \mathrm{mM} \mathrm{MgCl}, 11 \mathrm{mM}$ glucose; $\mathrm{pH}: 7.2$.

"Test B": $145 \mathrm{mM} \mathrm{NaCl}, 21 \mathrm{mM} \mathrm{KCl}, 0.7 \mathrm{mM} \mathrm{CaCl}, 0.7 \mathrm{mM} \mathrm{MgCl} 2,11 \mathrm{mM}$ glucose; $\mathrm{pH}: 7.2$.
Time dependence of the drug specific IL- 6 release

Typical time dependence of IL-6 release upon drug challenge of PBMCs of a hypersensitive person is shown in Figure 2, demonstrating that the 20-minute incubation time resulted in maximal release.

\section{IL-6 release from PBMCs incubated for $\mathbf{2 0}$ minutes with different drug concentrations}

The average IL-6 release increased significantly over the background level at all tested drug concentrations only in the positive test series. The mean increases of IL-6 release were $75 \%$ at $0.15 \mu \mathrm{M}, 69 \%$ at $0.25 \mu \mathrm{M}, 103 \%$ at $0.35 \mu \mathrm{M}$ and $96 \%$ at $0.5 \mu \mathrm{M}$ final concentrations of various drugs (Figure 3). Both the highest mean cytokine release and the highest number of positive results were found at $0.35 \mu \mathrm{M}$ drug concentration in Test $A$ medium. In Test $B$ medium the highest IL- 6 release and the highest number of positive results were detected at $0.15 \mu \mathrm{M}$ and at $0.25 \mu \mathrm{M}$ drug concentrations, respectively. In negative test series and in controls the average IL-6 release was not significantly different from that of the diluents at any drug concentrations tested. LDH concentrations were low (1-3 U/l) both for the positive and negative cases proving the presence of intact cells. Those samples containing damaged cells upon separation (LDH concentration 130-150 U/l) were excluded from further evaluation.

\section{Reliability of IL-6 release measurements in "Test $A$ " and "Test $B$ " groups}

Table 3 summarizes the results at 10 controls and 50 patients of Test A compared to 12 controls and 36 patients of Test $B$ groups tested in vitro -in vivo simultaneously. The parallel tests varied between 1 and 5 per individual persons. Some patients were tested in vivo by different drugs or with the same drug using different tests. In group A there were 99 tests out of which 13 in controls, and 70 in the patients gave identical results. Among concordant positive tests there were $63 \%$ mild systemic reactions in 25 instances due to oral provocation and in one case as the complication of positive patch testing. In 2 patients anaphylaxis (grade II) occurred upon intravenous administration of ferric sodium gluconate. IL-6 release was later positive to 3 differently colored monocomponent ferric oxides yielding thus $2 \times 3$ matching results. In this group $20 \%$ identical patch tests and $17 \%$ intradermal tests were obtained. Among matching negative tests $69 \%$ provocation $9,5 \%$ patch and $21,5 \%$ intradermal were noted. Within "Test $B$ " group there were 85 tests out of which 19 in the control group and 49 in the patient group gave identical results. One in vitro test was false positive but this person used inhalant steroid for asthma while tolerating ropivacain the substance, tested. 


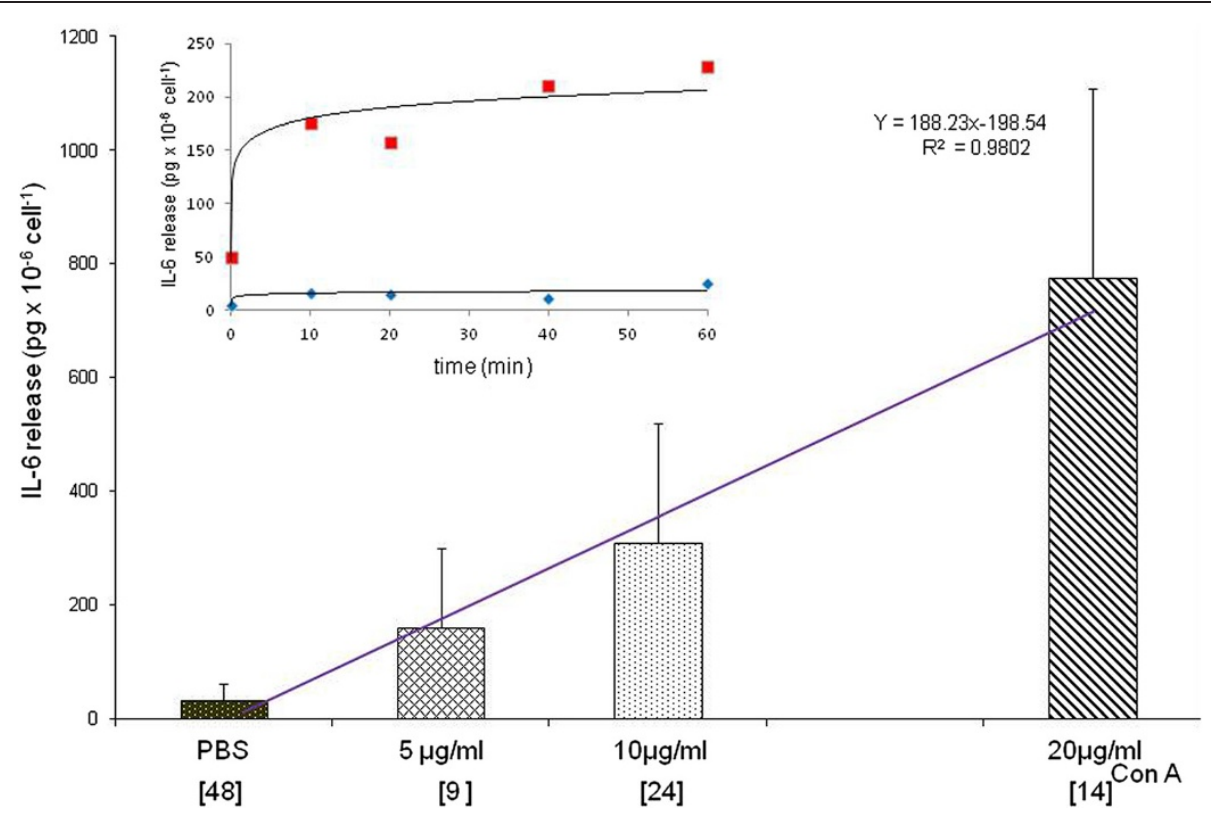

Figure 1 Effect of ConA on the release of IL-6 from mononuclear cells of patients after 20-min incubation with "Test A" solution. The columns represent mean \pm SD. [Brackets under abscissa indicate numbers of tests at different concentrations]. The value of $2197 \pm 268 \mathrm{pg} \times 10^{-6}$ cells ${ }^{-1}$ was obtained at $300 \mu \mathrm{g} / \mathrm{ml}$ ConA. Insert: fitted time course of the mean values from 2 independent experiments using $5 \mu \mathrm{g} / \mathrm{ml}$ ConA on 2 non-allergic persons' cells (red: ConA, blue: PBS).

Among concordant positive tests $43 \%$ were oral, sc or iv provocations, $14 \%$ patches and $43 \%$ intradermal ones. Out of the matching negative results $49 \%$ were due to provocation, $13 \%$ due to patch testing and $37 \%$ intradermal testing. The non-matching tests are marked with asterisks in Table 3. The in vitro test sensitivity in group "Test A" was markedly higher than in "Test B" (85.4\% versus $44.4 \%)$. In contrary, the test specificity was higher in the "Test B" group (93.1\%) than in the "Test A" (82. 4\%). However, both overall reliability and predictive values were higher in the "Test $A$ " than in "Test B" group.

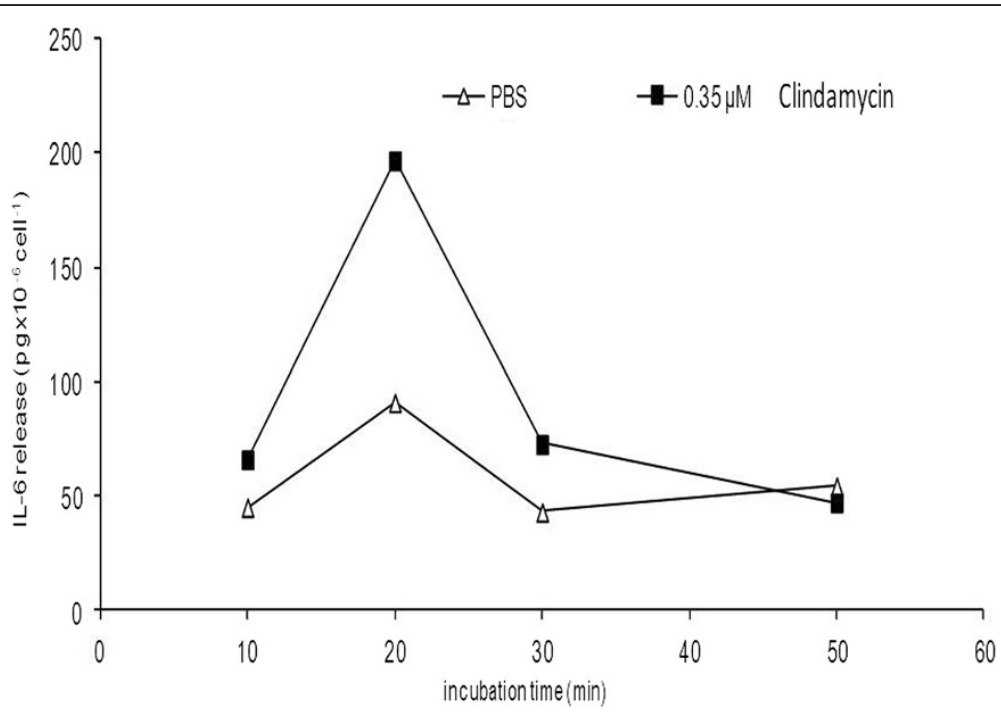

Figure 2 Time course of drug-induced IL- 6 release from PBMCs of an allergic patient (clindamycin, phenotype ANO) 6 months after the event. The drug was taken orally as monotherapy. Localized edematous rash has developed around the wrist and on dorsa of hands $30 \mathrm{~min}$. after repeated intake. Results of 2 independent experiments with 6-week interval yielded 196 and $198 \mathrm{pg} \times 10^{-6}$ cells at $0.35 \mu \mathrm{M}$ (the points coincide). 


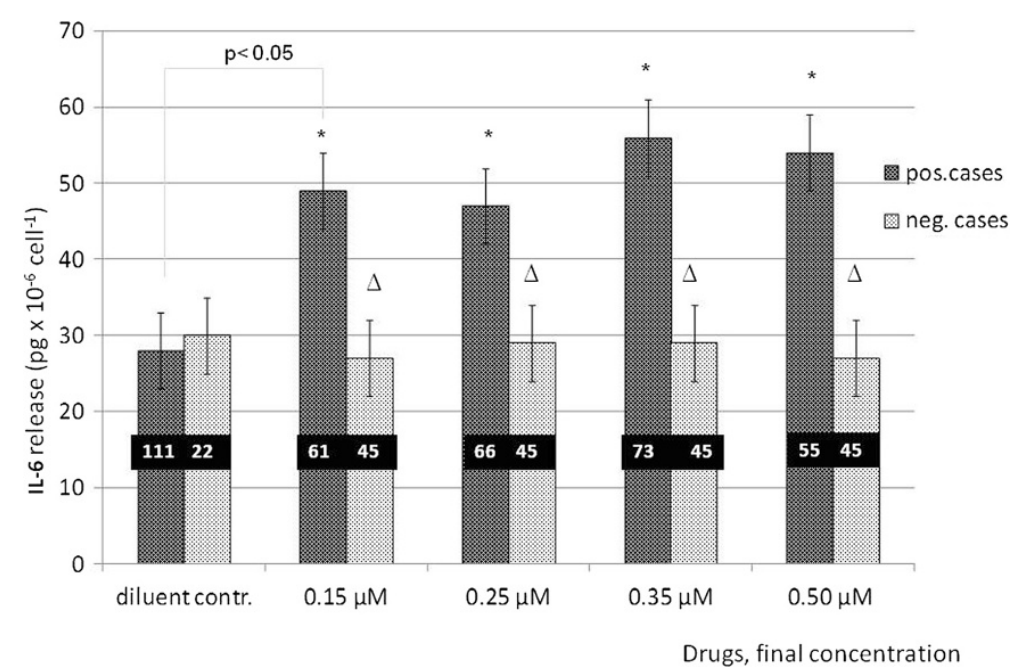

Figure 3 IL-6 release from PBMCs upon different drug concentrations of various drugs in positively and negatively reacting groups of the cohort incubated with "Test $A$ " medium. Positivity: $>50 \%$ increase in IL-6 release at any drug concentration relative diluent control. Stars indicate statistically significant differences) between negative control samples and positively tested cases, $(p<0.05)$ and between negative and positive cases $(p<0.005)$.

Distribution of the pharmacological classes of the tested drugs in the patients and in the control groups

The two dominant classes were antibiotics and nonsteroidal anti-inflammatory drugs (Figure 4a-b) in both test series. According to the individual histories 16 drug classes were tested both in the ADR-suspect groups and in the matching controls. Among the additives, iron

Table 3 Evaluation of the parallel in vitro-in vivo tests in the two groups

\begin{tabular}{lcccc}
\hline Parameters & $\begin{array}{c}\text { "Test } \boldsymbol{A}^{\text {" }} \\
\text { solution }\end{array}$ & $\%$ & $\begin{array}{c}\text { "Test } \boldsymbol{B}^{\text {" }} \\
\text { solution }\end{array}$ & \% \\
\hline Total N of parallel tests & 99 & & 85 & \\
Both negative & 42 & & 54 & \\
Both positive & 41 & & 12 & \\
Neg. IL-6, pos. in vivo* & 7 & & 15 & \\
Pos. IL-6, neg. in vivo** & 9 & & 5 & \\
Sensitivity & & 85.4 & & 44.4 \\
Specificity & & 82.4 & & 93.1 \\
Reliability & & 83.8 & & 77.6 \\
Positive predictive value & & 82.0 & & 70.6 \\
Negative predictive value & & 85.7 & & 78.2 \\
\hline
\end{tabular}

${ }^{*}$ false negative.

${ }^{* *}$ false positive.

"Test A": $100 \mathrm{mM} \mathrm{NaCl}, 24 \mathrm{mM} \mathrm{KCl}, 10 \mathrm{mM} \mathrm{CaCl} 2,10 \mathrm{mM} \mathrm{MgCl}, 11 \mathrm{mM}$ glucose; $\mathrm{pH}: 7.2$.

"Test B": $145 \mathrm{mM} \mathrm{NaCl}, 21 \mathrm{mM} \mathrm{KCl}, 0.7 \mathrm{mM} \mathrm{CaCl}, 0.7 \mathrm{mM} \mathrm{MgCl}, 11 \mathrm{mM}$ glucose; $\mathrm{pH}: 7.2$.

*in vivo: "Test $A$ ": oral provocation 2, intradermal 5 tests were positive.

*in vivo: "Test $B$ ": oral provocation 5 , epicutaneous 2 , intradermal 8 tests were positive.

**in vivo: "Test $A$ ": oral provocation 5, epicutaneous 1, intradermal 3 tests were negative.

**in vivo: "Test $B$ ": oral provocation 3, intradermal 2 tests were negative. oxides (E172) used to stain tablets were most often tested, and both positive and negative results were obtained. Sixteen additional drugs, among them enalapril (ANO and cough in history) gave only negative results. Some biologicals and cytostatic agents could not be evaluated although their molecular mass fell within the test range. In addition to drugs purified endotoxin (lipopolysaccharide) was tested in two independent experiments using serial dilutions. No additional IL-6 release exceeding PBS controls was detected.

\section{Clinical diagnoses and positive IL- 6 release at different standard drug concentrations}

The results for Test $A$ medium are listed in Table 1 . The relative frequency of single IL- 6 positivity within the test series is shown in columns I, II, III, while those with multiple positive IL-6 release appear in column IV. Comparing data of Table 1 with those in Figure 3, positive results comprised $61 \%(37 / 61)$ at $0.15 \mu \mathrm{M}, 38 \%$ $(25 / 66)$ at $0.25 \mu \mathrm{M}, 62 \%(45 / 73)$ at $0.35 \mu \mathrm{M}$ and $69 \%$ $(38 / 55)$ at $0.5 \mu \mathrm{M}$ drug concentrations, respectively. The number of tests with more than one drug concentration causing positive IL- 6 release was 65 out of a total of 153 positive tests $(42,5 \%)$. These results reflected the more widespread and severe skin and mucosal lesions of ADR (except for DRESS in one case). Using " $Z$ " test, combined data of lines 1,2,4,6,7,9 (column IV Table 1) were compared with those of lines 5,10-14 respectively, of column III representing less severe localized forms of ADR. The binomial distributions were significantly different $(\mathrm{p}<0.001)$ unlike in columns I and III where no significant differences were found. Multiple IL-6 

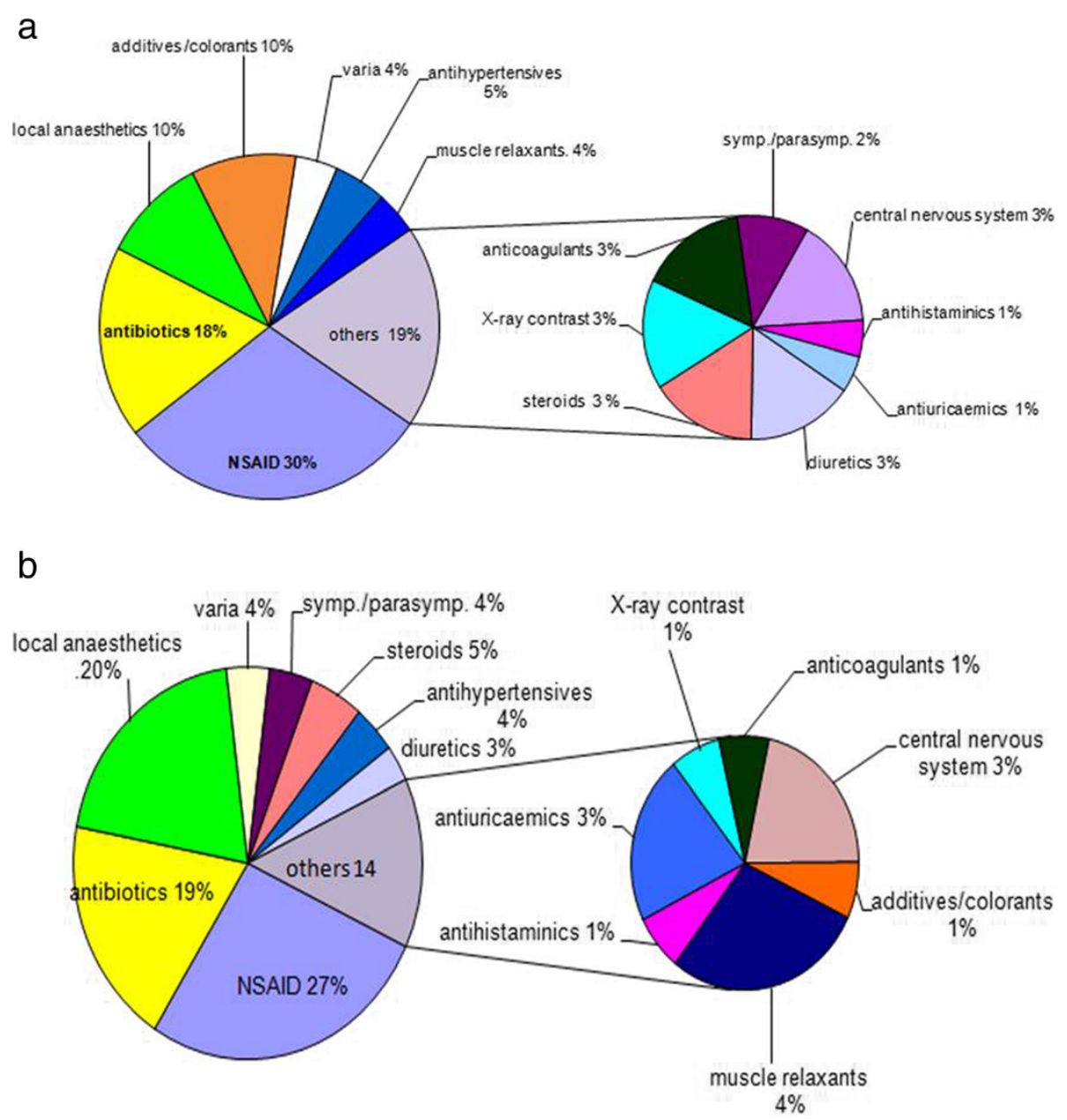

Figure 4 Distribution of drugs among different pharmacological classes. a: eliciting positive IL-6 release test results $(n=43)$. $\mathbf{b}$ : tested within the control group $(n=40)$. The numbers of individual drugs tested are higher $(\sim 70)$. Glibenclamide peripheral vasodilators and negative tests with acetylcystein are listed among "varia".

releases differentiated light from severe or widespread manifestations. These skin injuries (lines 1-4, 6-9; including a case of DRESS but only with culprit drug) and light and circumscribed ones differed significantly ("Z" test, $\mathrm{p}<0.05)$ in terms of drug concentrations eliciting maximal IL-6 release (column I). These severe generalized manifestations caused mostly positive IL- 6 release at the lowest drug concentration tested. Identical lines (5, 10-14) of columns I, III against IV were compared by $X^{2}$ test. No significant differences were found. Single peak positivities at $0.35 \mu \mathrm{M}$ (column II) showed a "mixed" pattern; both widespread severe and localized milder forms were represented here.

\section{Simultaneous release of IL-6, TNF-a (Figure 5) and IL-10}

Four patients with altogether 8 drugs and two control donors with exclusion of all types of ADR and negative oral provocations were tested. Concentrations of TNF- $\alpha$ and IL- 6 were simultaneously determined by the human
$\mathrm{Th}_{1} / \mathrm{Th}_{2}$ cytokine kit, together with IL-2, IL-4_ and IFN- $\gamma$ from the 20-minute supernatants of the PBMCs incubated with drugs or medium (Test $B$ ). There have been no measurable amounts of IL-2, IL-4 and IFN- $\gamma$ in any of the 10 test series. TNF- $\alpha$ and IL- 6 were present though. In six tests with negative IL-6 results PHA stimulation resulted in lowered TNF- $\alpha$ and increased IL-6 releases (Figure 5). Both control cases and tests of patients with nonreactive drugs as judged by their low IL- 6 release exhibited high TNF- $\alpha$ output. In cases where IL- 6 release test was positive the TNF- $\alpha$ release was significantly lower than in negative cases at all drug concentrations. In positive cases the highest IL-6 release was at $0.15 \mu \mathrm{M}$ drug concentration (Figure 6a). This opposite behavior in the release of the two inflammatory cytokines can even better visualized in relation of their own background (diluents) values (Figure 6b).

IL-10 and IL-6 were simultaneously measured in 13 tests of 6 donors in the 20-minute supernatants. Although 


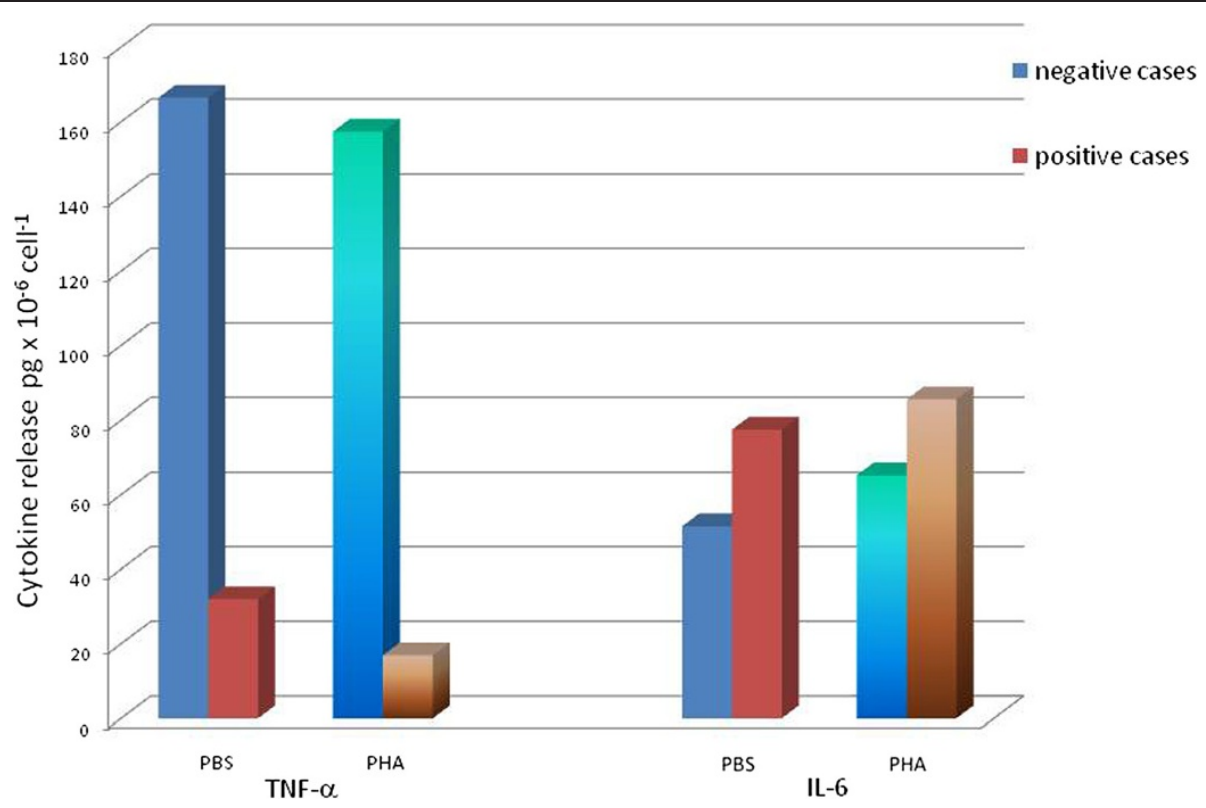

Figure 5 Mean cytokine releases stimulated by PHA-P $(168 \mu \mathrm{g} / \mathrm{ml})$ after 20-min incubation with "Test B solution" compared to controls. Non-allergic test series $(n=6)$ appear for both cytokines, TNFa and IL-6 in blue, allergic test series $(n=4)$ in light brown color.

upon challenge with $\mathrm{PHA}_{1}$ all donors' $\mathrm{PBMCs}$ released both IL-10 (80 pg/ml in average) and IL-6 (110 pg/ml in average) no detectable amount of IL-10 was released either in cases of positive (4 different drugs) or negative IL-6 responses (not shown in details).

\section{Discussion}

To obtain positive controls for the IL-6 release from PBMCs we used PHA exceeding about 20 times the amounts of those claimed to stimulate lymphocyte proliferation in 3-5-day cultures in the presence of serum [18]. On the other hand ConA was active in the same range as in LTT and increased the release of IL-6 in a dose- and time dependent manner (Figure 1). However, considerable inter- and intraindividual variations were experienced. Con A $(5 \mu \mathrm{g} / \mathrm{ml})$ seemed to act as proper positive control. PHA in $168 \mu \mathrm{g} / \mathrm{ml}$ concentration resulting in only $1-5 \%$ release of the expected preformed IL-6 from PBMC suspensions has acted in a similar limited fashion as the culprit drugs within the selected range. The question arises whether a small fraction of cells could account for the $1-5 \%$ of IL-6 release from a much larger $\left(>2000 \mathrm{pg} \times 10^{-6} \mathrm{cell}^{-1}\right)$ intracellular pool in $\mathrm{T}$ - lymphocytes as concluded from the extended dose response results obtained with Con A $(2197 \pm 268 \mathrm{SD}$ at $300 \mu \mathrm{g} / \mathrm{ml}$ upon 6 experiments $)$ or had the release occurred uniformly.

The time course of IL- 6 release upon drug challenge of PBMCs suggests that IL- 6 has originated from a preformed pool. The timing of the earliest onset of IL-6 synthesis was addressed by McHugh et al. [19]. They demonstrated that PHA has initiated de novo IL-6 production in PBMCs both of atopic and control donors after 4 hours. The maximal amount approximated $22-36 \times 10^{3} \mathrm{pg} / \mathrm{ml}$. Thus indirect evidence suggests a preformed pool size of one tenth of this magnitude.

The search for a more rapid and less cumbersome test replacing LTT in the diagnosis of a wide range of drug hypersensitivities has resulted in the detection of CD69 up regulation on a small group of CD4+ T-cells after 48 hrs of incubation [20]. The results are in good agreement with our findings.

IL-6 secretion has dropped in damaged cell suspensions (LDH increased in the supernatants) regardless of mitogen or to any drug concentration. Under "usual" assay conditions LDH was at detection limit. Thus, cytokine release due to cellular damage or to direct drug toxicity appears unlikely. Recent results on mouse mast cells have proven that specific desensitization of the animals either to ovalbumin or dinitrophenol blocked both the TNF- $\alpha$ and IL-6 releases from cells upon 30-minute and 4-hour in vitro challenges [12].

Comparing the in vitro vs. in vivo data for groups tested by Test $A$ or Test $B$ incubation media (Table 3 ) the importance of the signaling process became evident. In the early phase of these studies Dulbecco's rather simple solution enriched with $11 \mathrm{mM}$ glucose and supplemented with low concentrations of divalent cations $(0.7 \mathrm{mM}$ $\mathrm{Ca} 2+$ and $\mathrm{Mg} 2+$ ) was used in order to avoid cell-clumping [15]. The low test sensitivity shed light on the importance optimizing assay conditions. Raising the concentrations of $\mathrm{Ca}^{2+}$ and $\mathrm{Mg}^{2+}$ by fifteen-fold within the test medium resulted in a shift of the maximal IL- 6 release from 0.15 

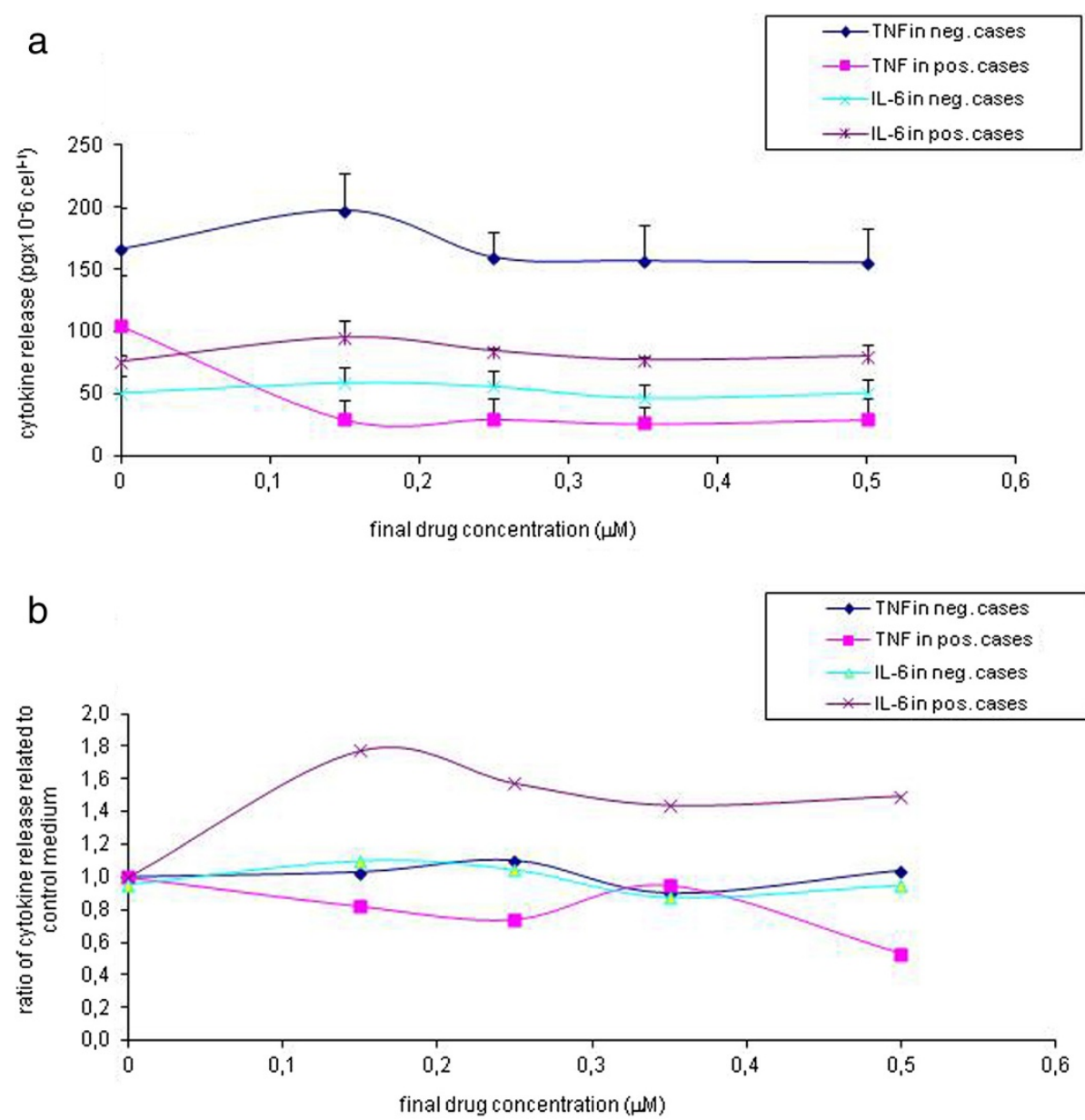

Figure 6 TNFa and IL- 6 release from PBMCs incubated with "Test B solutions" elicited by different drug concentrations measured by the CBA Th1-Th2 cytokine kit in a total of 6 negative and 4 positive assays. a: cytokine concentrations (mean +- S.E.M.); b: relative cytokine release normalized individually by their corresponding control values.

and 0.25 to 0.35 and $0.5 \mu \mathrm{M}$ (Figure 3). In Test $B$ the increase of IL-6 release was only 5\% compared to $103 \%$ and $96 \%$ obtained in Test $A$ medium at 0.35 and $0.5 \mu \mathrm{M}$, respectively. The concentrations accounting for test positivity were as follows: $0.15 \mu \mathrm{M}, 9$ cases, $0.25 \mu \mathrm{M}, 6$ cases, $0.35 \mu \mathrm{M}, 5$ cases and $0.5 \mu \mathrm{M}, 7$ cases. Using Test $B$ only 5 out of 122 tests had multiple positive readings against 65 out of 151 obtained with Test A (Table 1). The lack of IL-6 release at six out of the overall 13 false negativities could be attributed to the low $\mathrm{Ca}^{2+}$ and $\mathrm{Mg}^{2+}$ at 0.35 and $0.5 \mu \mathrm{M}$ drug concentrations. This means that the test sensitivity (against in vivo results) depends on the proper divalent cationic concentrations.

In our diagnostic groups there were both non-widespread and not life-threatening eruptions together with some serious and potentially lethal reactions (anaphylaxis grade II-III, DRESS). Beyond drugs most of them could have been caused by other elicitors too e.g. by infections $[3,21,22]$. Generalized disseminated dermatitis was clinically different from MPE. Stasis dermatitis of the legs is often aggravated by sensitization to drugs. Both toxic epidermal necrolysis, or acute generalized erythematous pustulosis (AGEP) have been tested earlier, but not with the standard media " $A$ " or "B". Thus, results were not included in Table 1. Multiple positive results with drugs suspected have been obtained though.

The concept of using multiple drug concentrations instead of only one was crucial to establish significant positive correlation between the severity (although not scored) and skin area involvement in most drug hypersensitivity related clinical phenotypes (Table 1), which had previously not been proven by any tests $[5,6,22]$, but were suggested by the 20-minute chromatin activation results $[1,9]$. Using molar concentrations, enables one to compare clinical manifestations elicited by chemically different drugs (between 76 and 4000 DA) since the number of tested molecules reacting with cellular receptors are identical. The receptor equivalence is also concordant with the $\mathrm{p}$-i concept of Pichler [4]. The rationale for selecting the lowest and the highest concentrations from 
the dilution series was to prove the inverse correlation between the drug concentration resulting in maximal IL-6 release and the severity of clinical reaction but only with the culprit drug. For the generalized widespread lesions the single peak positivity frequencies at $0.15 \mu \mathrm{M}$ are close to those of multiple positivity by comparing column I with column IV in Table 1. Our DRESS syndrome case e.g. with multivalent drug hypersensitivity had highly elevated IL-6 release with the relevant culprit drug at $0.15 \mu \mathrm{M}$, whereas another non-culprit drug (by history) has caused the peak exclusively at $0.5 \mu \mathrm{M}$. The in vivo tests are known to have different sensitivities and only provocations are considered as gold standards. Their percentages to evaluate any in vitro tests are important. The ratios of provocations were higher in Test A group than in Test B. Their use was not restricted only to prove negativity of in vitro tests. In many patients in vivo tests were performed successively starting with patch tests followed by intradermals which we attempted to standardize as well [23]. Our data have revealed that provocations against intradermal tests with $10^{-3} \mathrm{M}$ drug solutions had 33\% less positive results.

Recent results obtained on abacavir reactive CD8+ T-cell clones isolated from genetically susceptive HLA-B*5701+ individuals showed that their TCR exerted different avidity. Some of them reacted instantly to the drug in solution [24].

Our tests with methothrexate were highly positive at all concentrations in 3 treated rheumatological patients after widespread rashes. The same test resulted in false positivity in the two controls (who never took this antimetabolite before) at least in one drug concentration. LTT results were not satisfactory with this drug, either [25]. We recommend to perform IL-6 release assays with cytostatics emerging from the patients' history but keeping in mind that no published data are available yet. A possible candidate could be azathioprin [26]. For some biologicals (heparin and derivates) the test was proven of value [27] but the lack of experience with receptor antagonists, cytokine therapies and especially with high molecular weight proteins should be emphasized. The negativity of enalapril in 2 suspect cases reacting with cough and swelling points to the fact that in subjects with idiosyncrasy to ACE inhibitors none of the usual allergic mechanisms appear to be involved, therefore these drugs should be excluded from the tests ${ }^{\mathrm{a}}$.

The immunological synapse concept has emerged in recent years [28]. This might explain local signaling as early as 15 minutes after the onset of a close cell to cell contact in response to $1 \mu \mathrm{M}$ antigenic peptide as observed by total internal reflection microscopy or suggested by our earlier studies on chromatin birefringence changes using polarized light microscopy $[1,5,9]$. The $\alpha$-chain of IL-6 receptor binds both the soluble and membrane bound forms of its ligand. It is unable however, to induce signaling by itself. Trans-signaling occurs if gp 130, another membrane constituent binds to IL-6R $\alpha$. This may help to extend IL-6 stimulation to cells that lack IL-6 receptors but contain gp 130 [29]. IL-6/sIL-6R complexes regulate the inflammatory state, e.g. by inhibition of TNF- $\alpha$ [30]. In those early experiments in which exogeneous IL-6 was introduced to humans, induction of both IL-1R $\alpha$ that bound IL-1 $\beta$ and circulating TNF receptors was shown [31]. These factors might switch off the early apoptosis induced by certain drug concentrations, thus possibly being responsible for tolerance as well [32]. This cytokine antagonism might be inferred on drug specific cytokine release from the results demonstrated in Figures 5 and 6 as well. From earlier experiments of PBMCs in drug hypersensitive patients. a basic release of 100-300 pgxml $^{-1}$ TNF- $\alpha$ was evident at 24 hrs [2]. There are no data available for the time interval between 0-60 min. The authors have shown a time dependent decrease of TNF- $\alpha$ at 48 and 72 hours in unstimulated samples but inconsistent data for the culprit drugs of the sulfonamide as compared to aminopenicillin drug antigens have emerged. Similarly, the positive control (5 $\mathrm{\mu gxml}^{-1}$ tetanus toxoid), used has resulted in a tenfold drop in aminopenicillin sensitized patients' TNF- $\alpha$ releases against unsignificant elevation at sulfonamide allergic ones from 24 to $72 \mathrm{hrs}$ [2]. Our results point to an antagonism between the two early inflammatory cytokines. This seemed to be specific and concentration dependent with marked differences between sensitizing and tolerated drugs. Moreover, the direction of the changes in cytokine releases due to positive control polyclonal mitogen PHA and specific sensitizing drugs was the same. These results would need further corroborative studies, though.

Our data support the view that sensitivity to a given drug may well be reflected and quantified by the "early" IL- 6 release from patients' PBMCs. Thus, we suggest to measure as an appropriate rapid in vitro test, IL-6 in the supernatants of PBMCs stimulated with the "suspected" drugs with concentrations comparable on molar basis. The heterogeneity of the definition of positive drug allergy (positive response in drug patch test, or intradermal test, or drug provocation test) could be a possible weakness of the study.

\section{Endnotes}

${ }^{\mathrm{a}}$ In addition to Enalapril the following drugs gave only negative results: Acetylcystein, Ambroxol, Betaferon, Budesonid (2; epicutaneous test pos. in one) Chloropyramin, Drotaverin (3;1 false pos. in a control person), Famotidin, Ioversol, Clarythromycin, Pentasa, Salbutamol, Sulfametoxasol (3), Triamcinolon, Tramadol, Urapidin.

Automated serum IL-6 testing systems failed to detect PBMC released IL-6 although the standards for ELISA were detected with excellent linearity. The results of Test $A$ medium were not influenced by lowering glucose concentration to $7 \mathrm{mM}$. 


\section{Abbreviations}

ACE: Angiotensin converting enzyme; ADR: Adverse drug reaction; AMX: Amoxicilline; BSA: Bovine serum albumin; CBA: Cytometric bead array; Con A: Concanavalin A; DMSO: Dimethyl-sulfoxyde; DRESS: Drug reaction with eosinophilia and systemic symptoms; EAACl: European Academy of Allergy and Clinical Immunology; EDTA: Ethylene-diamine-tetraacetic acid; ENDA: European Network for Drug Allergy; FACS: Fluorescence activated cell sorter; gp: Glycoprotein; IFN: Interferon; JAK/STAT: Janus kinase/signal transducer and activator of transcription; LDH: Lactate dehydrogenase; LTT: Lymphocyte Transformation test; MAPK: Mitogen-activated protein kinase; MEM: Minimal essential medium; MHC: Mean histocompatibility complex; O.D.: Optical density; PBMC: Peripheral blood mononuclear cell; PBS: Phosphate buffered saline; TCR: T cell receptor.

\section{Competing interests}

The authors declare that they have no competing interests.

\section{Authors' contributions}

B-BjM conceived the study, selected patients and controls, carried out all clinical work including in vivo tests and drafted the manuscript. SK and LS led and performed all laboratory tests and participated in statistical analíysis as well as in text revision and improvement. SS conceived Concanavalin A experiments and confirmed the reproducibility of in vitro results in a remote laboratory. He also contributed in text and figure revisions due to reviewers' demands. All authors read and approved the final manuscript.

\section{Acknowledgements}

Authors are indebted to Ildikó Kovács (Debrecen) for providing her results on IL-6 release of PBMC and of non-adherent cells. The invaluable help by Klára Rásky (Budapest) is greatly acknowledged. Our deep gratitude is expressed to Prof. K. Balogh (Boston, USA) for critical revision of the manuscript.

\section{Author details}

${ }^{1}$ Department of Dermatology, Medical Center of Hungarian Defense Forces, Podmaniczky u. 109-111, Budapest H-1062, Hungary. ${ }^{2}$ Department of Pathophysiology, Medical Center of Hungarian Defense Forces, Róbert Károly krt. 44, Budapest $\mathrm{H}-1134$, Hungary. ${ }^{3}$ Division of Clinical Immunology, University of Debrecen, Móricz Zs. u. 22, Debrecen 4032, Hungary.

Received: 15 March 2013 Accepted: 3 November 2014

Published: 9 January 2015

\section{References}

1. De Sousa-Pinto JM, Babo MJ: In vitro diagnosis of drug allergy. Assessment of the Chromatin Activation Test (CAT). Pharmacoepidem Drug Safe 1997, 6(Suppl 3):861-869.

2. Lochmatter P, Beeler A, Kawabata TT, Gerber BO, Pichler WJ: Drug specif ic in vitro release of

IL-2, IL5, IL-13 and IFN- $\gamma$ in patients with delayed type drug hypersensitivity. Allergy 2009, 64:1269-1278.

3. Hausmann O, Schnyder B, Pichler WJ: Drug hypersensitivity reactions involving skin. Handb Exp Pharmacol 2010, 196:29-55.

4. Pichler WJ: Delayed drug hypersensitivity reactions. Ann Intern Med 2003 , 139:683-693.

5. Halevy S, Cohen AD, Grossmann N: Clinical implications of in vitro druginduced interferon gamma release from peripheral blood lymphocytes in cutaneous adverse drug reactions. J Am Acad Dermatol 2005, 52:254-261.

6. Merk HF: Diagnosis of drug hypersensitivity: lymphocyte transformation test and cytokines. Toxicology 2005, 209:217-220.

7. Holub MC, Makó E, Dévay T, Dank M,Szalai C, Fenyvesi A, Falus A: Increased interleukin-6 levels, interleukin-6 receptor and gp130 expression in peripheral lymphocytes of patients with inflammatory bowel disease. Scand J Gastroenterol 1998, 228:47-50.

8. Heinrich PC, Behrmann I, Haan S, Hermanns HM, Müller-Newen G, Schaper F: Principles of interleukin (IL)-6-type cytokine signalling and its regulations. Biochem J 2003, 374:1-20

9. Baló-Banga JM, Barabás É, Merétey K: Chromatin changes and adverse drug reactions. Central East Eur Derm-Venereol Assoc (CEEDVA) Bulletin 2003, 5:4-14.

10. Baló-Banga JM: New in vitro cytokine release tests for in vitro diagnosis of drug allergies [abstract]. J Eur Acad Derm Vener 2004, 18(Suppl 2):55.

11. Spencer LA, Szela CT, Perez SA, Kirchhoffer CL, Neves JS, Radke AL, Weller PF: Human eosinophils constitutively express multiple Th1, Th2 and immunoregulatory cytokines that are secreted rapidly and differentially. J Leukoc Biol 2009, 85:117-123.

12. Sancho-Serra Mdel C, Simarro M, Castells MC: Rapid IgE desensitization is antigen specific and impaired early and late mast cell responses targeting FceRI internalization. Eur J Immunol 2011, 41:1004-1013.

13. Karch FE, Lasagna L: Toward the operational identification of adverse drug reactions. Clin Pharmacol Therap 1977, 21:247-254.

14. Bøyum A: Isolation of mononuclear cells and granulocytes from human blood. Scand J Clin Lab Invest 1968, 21(Suppl 97):77-89.

15. Dulbecco R, Vogt M: Plaque formation and isolation of pure cell lines with poliomyelitis viruses. J Exp Med 1954, 99:167-182.

16. Brailly H, Montero-Julian FA, Zuber CE, Flavetta S, Grassi J, Houssiau F, vanSnick J: Total interleukin-6 in plasma measured by immunoassay. Clin Chem 1994, 40:116-123.

17. Aberer W, Bircher A, Romano A, Blanca M, Campi P, Fernandez J, Brockow K Pichler WJ, Demoly P: Drug provocation testing in the diagnosis of drug hypersensitivity reactions: general considerations. Allergy 2003, 58:854-863.

18. Tabakov VU, Litvina MM, Schepkina JV, Jarilin AA, Chestkov W: Studying the proliferation of human peripheral blood T lymphocytes in serum-free medium. Bull Exp Biol Med 2009, 147:120-124.

19. McHugh SM, Wilson AB, Deighton J, Lachmann PJ, Ewan PW: The profiles of interleukin (IL)-2, IL-6, and interferon-gamma production by peripheral blood mononuclear cells from house dust-mite allergic patients: a role for IL-6 in allergic disease. Allergy 1994, 49:751-759.

20. Beeler A, Zaccaria L, Kawataba T, Gerber BO, Pichler WJ: CD69 upregulation on $\mathrm{T}$ cells as an in vitro marker for delayed type drug hypersensitivity. Allergy 2008, 63:181-188.

21. Mockenhaupt M: Epidemiology of cutaneous adverse drug reactions. In Adverse Cutaneous Drug Eruptions. Edited by French LE. Basel: Karger; 2012:1-17 [Chem Immunol Allergy, vol 97.].

22. Pirmohamed M, Friedman PS, Molokhia M, Loke YK, Smith C, Phillips E, La Grenade L, Carleton B, Papaluca-Amati M, Demoly P, Shear NH: Phenotype standardization for immune-mediated drug-induced skin injury. Clin Pharmacol Ther 2011, 89:896-901.

23. Baló-Banga JM, Vajda A: Attempts to standardize intradermal drug tests based on molecular mass and on clinical phenotypes. Some pitfalls or exceptions? Clin Transl Allergy 2014, 4(Suppl 3):102

24. Adam J, Eriksson KK, Schnyder B, Fontana S, Pichler WJ, Yerly D: Avidity determines T-cell reactivity in abacavir hypersensitivity. Eur J Immunol 2012, 42:1706-1716.

25. Hirata S, Hattori N, Kumagai K, Haruta Y, Yokoyama A, Kohno N: Lymphocyte transformation test is not helpful for the diagnosis of methotrexate-induced pneumonitis in patients with rheumatoid arthritis. Clin Chim Acta 2009, 407:25-29.

26. Mori H, Yamanaka K, Kaketa M, Tamada, K, Hakamada A, Isoda K, Jamanishi K, Mizutani H: Drug eruption caused by azathioprine: value of using the drug-induced lymphocytes stimulation test for diagnosis. J Dermatol 2004, 31:731-736

27. Jagicza A, Bata Z, Mihályi L, Kemény L, Kenderessy-Sz A, Schweitzer K, Baló-Banga JM: A case of heparine allergy. Börgyógy Vener Szemle [Hung] 2008, 84:76-79.

28. Krummel MF, Cahalan MD: The immunological synapse: a dynamic platform for local signaling. J Clin Immunol 2010, 30:364-372.

29. Jones SA, Richards PJ, Scheller J, Rose-John S: IL-6 transsignaling: the in vivo consequences. J Interferon Cytokine Res 2005, 25:241-253.

30. Igaz P, Horváth A, Horváth B, Szalai C, Pállinger E, Rajnavölgyi E, Tóth S, Rose-John S, Falus A: Soluble interleukin-6 receptor (sIL-6R) makes IL-6 negative T cell line respond to IL-6; it inhibits TNF production. Immunol Lett 2000, 71:143-148.

31. Tilg H, Trehu E, Atkins MB, et al: Interleukin-6 (IL-6) as an antiinflammatory cytokine: induction of circulating IL-1 receptor antagonist and soluble Tumor necrosis factor receptor p55. Blood 1994, 83:113-118.

32. Réthy LA, Baló-Banga JM: Drugs as haptens induce apoptosis in lymphocytes from patients with drug-allergies. In Proc. 14th European Immunology Meeting (EFIS 2000). Edited by Mackiewicz A, Kurpisz M, Zeromski J. Bologna: Monduzzi Editore S.p.A; 2001:105-112.

doi:10.1186/1939-4551-8-1

Cite this article as: Baló-Banga et al:: A novel rapid (20-minute) IL-6 release assay using blood mononuclear cells of patients with various clinical forms of drug induced skin injuries. World Allergy Organization Journal 2015 8:1. 